\title{
ARTICLE
}

\section{The effect of chronic oxytocin treatment during abstinence from methamphetamine self-administration on incubation of craving, reinstatement, and anxiety}

\author{
Nicholas A. Everett (iD), Sarah J. Baracz ${ }^{1}$ and Jennifer L. Cornish ${ }^{1}$
}

\begin{abstract}
Methamphetamine (METH) abuse is characterised by chronic relapse and anxiety, for which there are no effective pharmacotherapies. Acute treatment with the neuropeptide oxytocin has shown therapeutic potential for METH addiction and has social and anxiolytic effects in METH-naïve rats. However, the effects of chronic oxytocin treatment in METH-experienced rats are unknown. This study investigated the effects of repeated oxytocin treatment during abstinence from METH self-administration on incubation of cue-induced relapse, yohimbine- and METH-induced reinstatement, trait anxiety, and social interaction. Male and female Sprague-Dawley rats self-administered intravenous METH for $2 \mathrm{~h} /$ day (12 days) and then on short-access ( $2 \mathrm{~h} /$ day; ShA) or long-access ( $6 \mathrm{~h} /$ day; $\mathrm{LgA}$ ) sessions (10 days). Rats underwent 30 days of drug abstinence, during which they received 15 days of intraperitoneal oxytocin (1 mg/kg) or saline (days 6-20) injections. Anxiety and social interaction were tested on days $25-28$, and incubation was assessed by testing cue-induced relapse on days 2 and 30 . Rats underwent extinction after the final cue-relapse test, followed by yohimbine- and METH-primed reinstatement. LgA, but not ShA rats exhibited incubation of METH-craving and enhanced METH-primed reinstatement in both sexes, and enhanced yohimbine-induced reinstatement in females. Importantly, chronic oxytocin attenuated incubation and METH-primed reinstatement in both sexes, and yohimbine-induced reinstatement in females, although only in LgA rats. LgA produced a heightened anxiety phenotype, which was partially rescued by chronic oxytocin treatment. Using a translatable addiction model, these findings demonstrate the therapeutic efficacy of chronic oxytocin after METH self-administration and supports the clinical utility of oxytocin for METH addiction in both sexes.
\end{abstract}

Neuropsychopharmacology (2020) 45:597-605; https://doi.org/10.1038/s41386-019-0566-6

\section{INTRODUCTION}

Abuse of, and addiction to, the psychostimulant methamphetamine (METH) is a rapidly growing public health concern and is the second most abused illicit drug worldwide [1]. Methamphetamine addiction is characterised by chronic relapse, and often co-occurs with anxiety [2, 3], which may contribute to heightened relapse risk [4]. There are currently no approved pharmacotherapies which prevent relapse to METH addiction [5]. The neuropeptide oxytocin has shown substantial promise in the preclinical literature as a potent inhibitor of METH selfadministration [6-8], METH reward [9, 10], cue-induced relapse $[8,11,12]$, METH-induced relapse $[6,13-15]$, and yohimbineinduced relapse [7, 16]. The social [17] and anxiolytic [18] effects of oxytocin treatment may have added benefits for METHdependent individuals. Together, these promising findings from animal models have set the stage for the pre-registration of the first randomised, double-blind, placebo-controlled trial investigating the effects of oxytocin as an adjunct to psychosocial therapy in humans with a METH-use disorder [19]. However, clinical utility will require chronic treatment with oxytocin, the effects of which have not been widely investigated.

Within the preclinical addiction field, the effects of chronic oxytocin treatment have only been investigated prior to access to the addictive drug, and typically during a crucial developmental window. For example, rats treated for 10 days with oxytocin during adolescence exhibited reduced free-consumption of alcoholic beer [20] and reduced motivation to self-administer METH [21]. However, when administered to adult rats for 10 days prior to METH self-administration, oxytocin had no effect on reinstatement to METH-seeking behaviours unless the rats had experienced chronic stress [16]. The experience-dependent effects of chronic oxytocin indicate that the therapeutic utility of oxytocin may need to be understood in animal models exhibiting a robust addiction-like phenotype (for review see ref. [22]).

Intravenous drug self-administration procedures can be modified to induce distinct addiction phenotypes. For example, although procedures involving short daily access (ShA) to METH $(\sim 2 \mathrm{~h} /$ day) are widely used, this model does not necessarily induce human-like addiction traits. In contrast, long daily access $(\mathrm{LgA})$ to METH ( $\geq 6 \mathrm{~h} /$ day) can produce incubation of cue-induced craving during protracted abstinence [23], which is also reported in human METH users [24]. Other dysfunctions reminiscent of human METH addiction are also induced by $\mathrm{LgA}$, including memory impairments [25], deficient sensorimotor gating [26], dysphoria [27], and altered glutamate and dopamine neurotransmission $[28,29]$. Treatment with oxytocin interacts with many of these behaviors [30-32] and neurotransmitter systems [12, 33, 34] disrupted by $\operatorname{LgA}$ that may contribute to addiction, although the

${ }^{1}$ Centre for Emotional Health, Department of Psychology, Macquarie University, Balaclava Road, North Ryde, NSW 2109, Australia

Correspondence: Nicholas A. Everett (nick.everett@mq.edu.au)

Received: 29 July 2019 Revised: 27 October 2019 Accepted: 3 November 2019

Published online: 12 November 2019 
598

effects of oxytocin treatment have not yet been studied in these translational LgA models.

The present study investigated the effects of chronic oxytocin treatment when administered during a period of protracted abstinence from ShA or LgA METH self-administration, on cue-, drug-, and yohimbine-induced relapse, as well as on anxiety-like behaviours. Sex was included as a primary variable as sex interacts with both oxytocin (e.g. ref. [35]) and METH (e.g. ref. [36]), and acute oxytocin treatment interferes with METH behaviours differently between the sexes [7].

\section{MATERIALS AND METHODS}

Subjects

Pregnant Sprague-Dawley dams were purchased (Animal Resources Centre, Australia), and pups were weaned at postnatal day (PND) 22. A total of 48 male and 48 freely-cycling female offspring were pair-housed by sex in open-top plastic cages (lights on at 07:00, off at 19:00). Rats had ad libitum access to chow and water. All procedures were conducted in accordance with the Australian Code of Practice for the Care and Use of Animals for Scientific Purposes (8th Edition, 2013), and the Macquarie University Animal Ethics Committee.

\section{Drugs}

Methamphetamine hydrochloride was purchased (Australian Government Analytical Laboratories, Australia), and oxytocin was synthesized by ChinaPeptides (China). Yohimbine hydrochloride was purchased from Sigma Aldrich (Australia). For intraperitoneal (IP) injection, drugs were prepared in $0.9 \%$ physiological saline (oxytocin, METH, $1 \mathrm{ml} / \mathrm{kg}$ ) or distilled water (yohimbine, $2 \mathrm{ml} / \mathrm{kg}$ ). For self-administration, METH was dissolved in saline at $0.1 \mathrm{mg} / \mathrm{kg}$ per $50 \mu \mathrm{l}$ infusion. METH was prepared every 1-2 days for each sex, based on their average body weight.

\section{Surgery}

Rats (PND 47) were randomly assigned to a METH-naïve group (16/ sex) or were implanted with jugular vein catheters for intravenous METH self-administration (32/sex). Surgical and post-operative procedures were performed as previously described [14], after which self-administration procedures began in early adulthood (PND 56).

\section{Experimental procedures}

Experimental groups and timeline of procedures is depicted in Fig. 1. For each test, descriptions of apparatus are included in the Supplementary Materials and Methods.

Methamphetamine self-administration and extended access Rats lever pressed for METH during daily fixed-ratio- 1 sessions across 12 consecutive days $(2 \mathrm{~h} /$ day $)$ in operant chambers. Depression of the active lever resulted in an intravenous METH infusion, illumination of a cue light for 3-seconds, and 20-seconds without the house light, signaling a time-out period where METH was unavailable. Following the 12 th self-administration session, rats were randomly allocated to either continue self-administration for $2 \mathrm{~h}$ (Short Access, "ShA"), or $6 \mathrm{~h}$ per day (Long Access, "LgA") for 10 days. Allocated ShA and LgA groups did no differ regarding: body weight, total METH intake, or rate of acquisition (data not shown). Infusions were limited to a maximum of 60 (ShA) and 150 (LgA) per session, otherwise ShA and LgA were identical.

Treatment during forced abstinence from methamphetamine Following the final day of self-administration, rats underwent 30 days of forced abstinence where they remained in their home cages. Rats were randomly allocated to receive oxytocin $(1 \mathrm{mg} / \mathrm{kg}$ IP) or saline injections on days 6-20 of abstinence. Allocations were conducted by home cage, so that each pair received the same treatment. Treatment groups did not differ regarding total METH intake, body weight, or lever pressing on cue-extinction day 2 .

\section{Elevated plus maze}

On the 25th abstinence day, rats were placed in the centre of the elevated plus maze (EPM) facing an open arm and could freely explore the apparatus ( $5 \mathrm{~min}$ ). Time spent in the open arms was scored by a person blind to treatment group. For both EPM and social interaction test, rats with no METH exposure but which had received 15 days of oxytocin or saline were also assessed.

\section{Social interaction test}

On the 26th abstinence day, rats were individually habituated to the testing arena (20 mins), and then on abstinence days 27-28 they were tested for their social interaction with novel partners. Each rat separately interacted with two novel conspecifics of their same group (METH-Access; Sex; Chronic-Treatment), and body weights of pairs were within $10 \%$. Interaction pairs were not siblings and were from nonadjacent home cages. Pairs of rats were allowed to interact for a 5-minute period. The time spent engaged in social behaviours was scored by a researcher blind to treatment condition. Measurement of social behaviour was based on [20], and included: anogenital investigation, nose-to-nose investigation, pinning, and climbing, which were scored as one aggregate variable.

Incubation of methamphetamine craving

To model incubation of METH craving, rats were returned to operant chambers on forced abstinence days 2 and 30 and underwent extinction tests in the presence of methamphetamineassociated cues (for review, see [37]). During these sessions, rats were exposed to a single illumination of the cue-light 30 seconds into the session. Thereafter, depression of the active lever illuminated the cue light for 3 seconds. The difference in active lever pressing from day 2-30 was calculated to create an "Incubation Score" [38].

\section{Extinction of lever pressing}

After the cue-extinction test on day 30 , rats underwent daily 1-h sessions to extinguish lever pressing, where depression of either lever had no programmed consequences. Following 8 days of extinction, all rats met standard extinction criterion of $<15$ active lever presses. Rats received saline injections (IP) immediately prior to the final 2 sessions, to habituate them to reinstatement conditions.

Yohimbine- and methamphetamine-primed reinstatement Following extinction, rats underwent yohimbine- and then METHprimed reinstatement to drug seeking. Yohimbine-induced reinstatement was achieved by $I P$ injection with the $a-2$ adrenoceptor antagonist yohimbine, which induces cravings in humans [39] and reinstatement in rodents [40]. Reinstatement tests were separated by three extinction sessions, and all rats met extinction criterion prior to the next reinstatement test. All yohimbine- and METH-primed reinstatement sessions were programmed identically to extinction sessions. On the first session, rats received $0.625 \mathrm{mg} / \mathrm{kg}$ yohimbine, were returned to their home cage for $30 \mathrm{~min}$, and then placed in their operant chamber for $1 \mathrm{~h}$. Reinstatement session two was identical, except rats received $1.25 \mathrm{mg} / \mathrm{kg}$ yohimbine. Reinstatement sessions three and four began $5 \mathrm{~min}$ after rats received 0.3 or $1.0 \mathrm{mg} / \mathrm{kg} \mathrm{METH}$, respectively.

Statistical analysis

Self-administration and reinstatement data were analysed using a between subject's ANOVA, including independent variables of METH-Access (ShA, LgA), Chronic-Treatment (saline, oxytocin), and Sex (male, female) for a total of eight groups ( $n=8 /$ group). Data from the social interaction test and EPM were analysed similarly, with the addition of a third level of METH-Access ("None") for a total of 12 groups ( $n=8 /$ group). For reinstatement data, inactive 


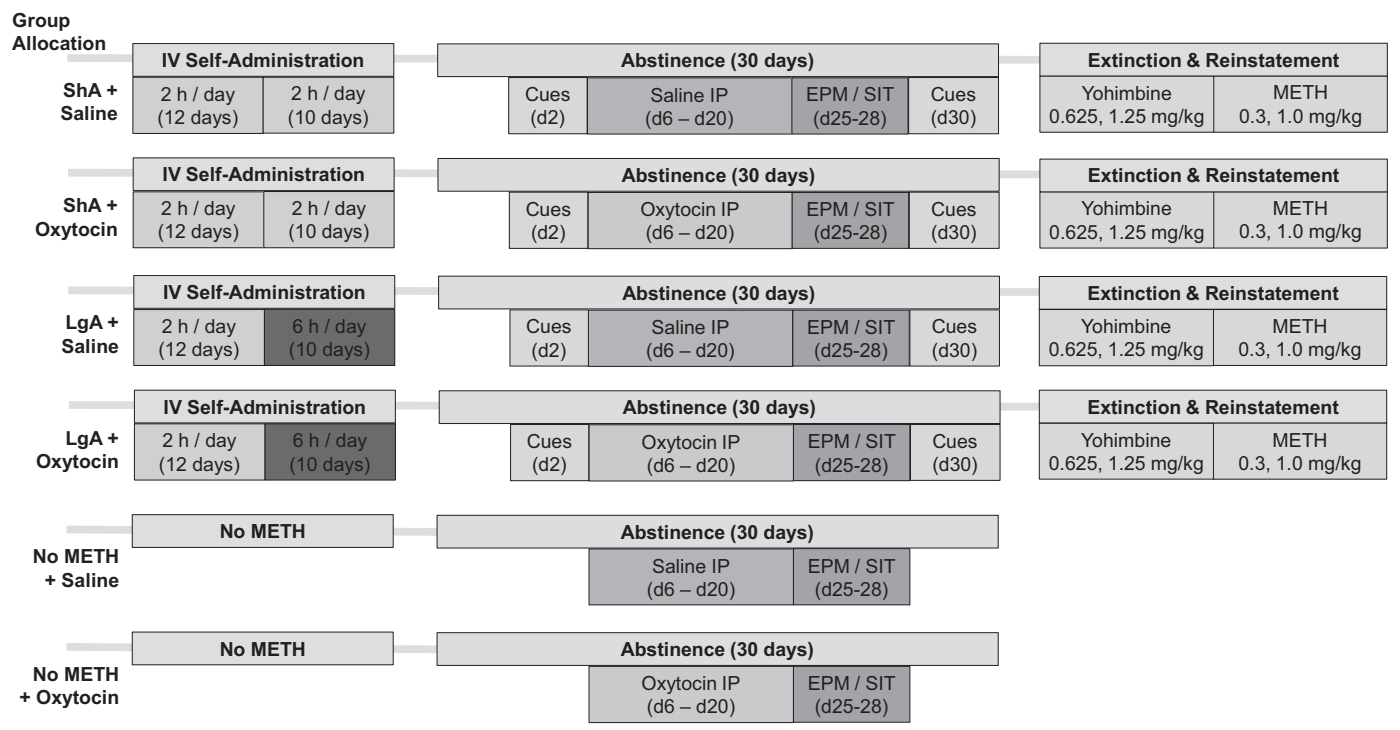

Fig. 1 Timeline of experimental procedures. ShA Short Access to METH self-administration ( $2 \mathrm{~h} /$ day). LgA Long Access to METH selfadministration ( $6 \mathrm{~h} /$ day). EPM Elevated Plus Maze. SIT Social Interaction Test. $n=8 /$ sex/group.

lever pressing was included as a covariate, and dose of yohimbine $(0.625,1.25 \mathrm{mg} / \mathrm{kg}))$ and METH $(0.3,1.0 \mathrm{mg} / \mathrm{kg})$ was included as a within-subjects variable. Main effects, interactions $(a=0.05)$ and simple effects adjusted with Bonferroni's test for multiple comparisons ( $a=0.05 / \#$ possible contrasts) are reported. Analyses were conducted in SPSS (IBM, Version 20), and visualized in Graphpad (Prism, Version 7.04). Analyses of inactive lever pressing and locomotor data is presented in the Supplementary Results.

\section{RESULTS}

Methamphetamine self-administration

All rats acquired METH self-administration (Fig. 2a). Extended access to METH self-administration ( $\mathrm{LgA})$ resulted in significantly higher life-time METH intake than ShA $(F(1,58)=274.933, p<$ 0.001 ; Fig. 2b). The binge-like intake at the start of sessions was enhanced by $\operatorname{LgA}$, whereby METH intake in the first 15 and first 120 min of the session was significantly higher after 10 days of $\operatorname{LgA}$ training, compared with rats first $\operatorname{LgA}$ session (all $p<0.01$; Fig. 2d). There was no significant effect of Sex on METH intake $(F(1,58)=0.092, p=0.866)$. There was a significant Sex $\times$ METHAccess interaction on locomotor activity, whereby females exhibited higher locomotor counts more in the $\operatorname{LgA}$ condition than ShA $(F(1,58)=107.32, p<0.001$; Fig. $2 c)$.

Effect of methamphetamine self-administration and chronic oxytocin treatment on anxiety

On the EPM test, the interactions of Sex $\times$ METH-Access $\times$ ChronicTreatment $(\mathrm{F}(1,84)=0.220 ; p=0.803)$, Sex $\times$ METH-Access $(\mathrm{F}(1,84)=$ $0.022 ; \quad p=0.978), \quad$ METH-Access $\times$ Chronic-Treatment $\quad(\mathrm{F}(1,84)=$ $1.470 ; \quad p=0.236)$, and Sex $\times$ Chronic-Treatment $(F(1,84)=0.254$; $p=0.616)$ on time spent in the open arm were not significant. There were significant main effects of METH-Access $(F(1,84)=9.221$; $p<0.001$; Fig. 3a), Chronic-Treatment $(\mathrm{F}(1,84)=5.410, p=0.022)$, but not Sex $(F(1,84)=0.787 ; p=0.377)$ on open arm time. Simple contrasts $(a=0.017)$ revealed that compared with METH-Naive rats, LgA Saline rats spent significantly less time in the open arms (Males: $p=0.009$; Females: $p=0.010$ ), while ShA Saline rats were not significantly different to METH-Naïve Saline (Males: $p=0.488$; Females: $p=0.240$ ). Comparing between METH-Access groups, $\mathrm{LgA}$ rats spent less time in the open arms than ShA, although this was not significant after controlling for multiple comparisons (Males: $p=0.040$; Females: $p=0.120$ ). Within LgA, the oxytocin group spent more time in the open arms than saline, although this too was not significant after controlling for multiple comparisons (Males: $p=$ 0.040; Females: 0.056).

Effect of methamphetamine self-administration and chronic oxytocin treatment on social interaction

On the social interaction test, there were no significant interactions of METH-Access $\times$ Sex $\times$ Chronic-Treatment $(F(1,84)=0.420$; $p=0.658$; Fig. 3b), METH-Access $\times \operatorname{Sex}(\mathrm{F}(1,84)=1.752 ; p=0.180)$, Sex $\times$ Chronic-Treatment $(\mathrm{F}(1,84)=0.613 ; p=0.436)$, or METHAccess $\times$ Chronic-Treatment $(\mathrm{F}(1,84)=0.300 ; p=0.742)$, on time spent interacting. There was a significant effect of $\operatorname{Sex}(F(1,84)=$ 12.749; $p<0.001)$, where overall, female pairs spent less time engaged in social interaction than male pairs. There was a significant effect of Chronic-Treatment $(F(1,84)=8.907 ; p=0.004)$, whereby oxytocin treated pairs engaged in more social interaction than saline treated pairs. There was a significant effect of METHAccess on social interaction time $(\mathrm{F}(1,84)=36.891 ; p<0.001)$, and simple contrasts $(a=0.017)$ revealed that compared with METHNaïve pairs, LgA pairs spent significantly less time interacting (Males: $p<0.001$; Females: $p=0.001$ ) while ShA pair interaction did not differ to naïve controls (Males: $p=0.516$; Females: $p=$ 0.236). LgA pairs also interacted significantly less than ShA pairs (Males: $p<0.001$; Females: $p=0.011$ ). Within LgA, oxytocin treated pairs did not differ significantly in time spent interacting than saline pairs (Males: $p=0.261$; Females: $p=0.110$ ). However, within METH-Naïve females, oxytocin treatment significantly increased social interaction time compared with saline pairs $(p=0.016)$.

Effects of chronic oxytocin treatment on incubation of cueinduced reinstatement

When comparing day 2 to day 30, all four LgA groups and ShA females significantly increased their active lever pressing over time (all $p<0.002$; Figure S2 and Table S2), while ShA males did not (Saline: $p=0.162$, Oxytocin: $p=0.060$ ). Incubation Scores (day 30-day 2) were therefore analysed to detect differences in magnitude of incubation. There were no significant interactions of Sex $\times$ METH-Access $\times$ Chronic-Treatment $(F(1,58)=0.033 ; p=$ $0.856)$, Sex $\times$ METH-Access $(F(1,58)=0.182 ; p=0.671)$, or Sex $\times$ Chronic-Treatment $(F(1,58)=0.232 ; p=0.632)$ on incubation scores. There was a significant interaction of METH-Access $\times$ Chronic-Treatment on incubation scores $(F(1,58)=11.151 ; p=$ 0.001 ), whereby the inhibitory effect of oxytocin versus saline on 


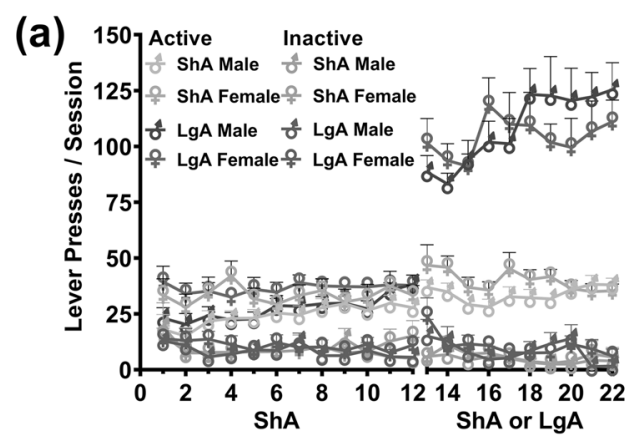

(c)

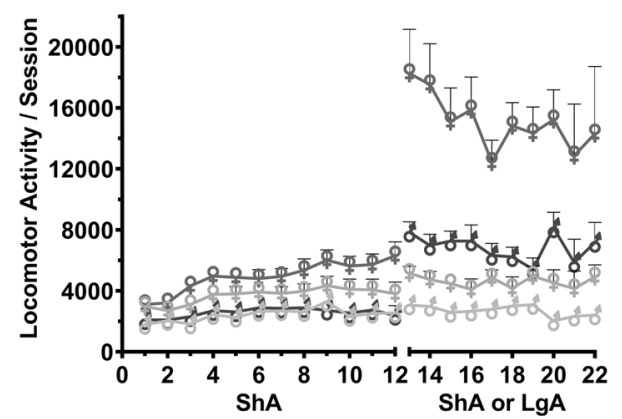

(b)

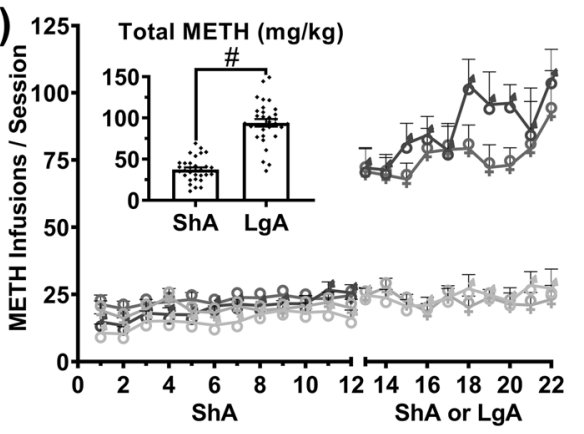

(d)

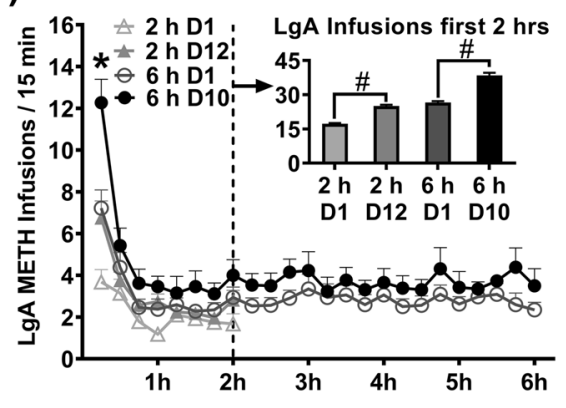

Fig. 2 Acquisition and escalation of METH $(0.1 \mathrm{mg} / \mathrm{kg} /$ infusion) intravenous self-administration during short access (ShA: $2 \mathrm{~h} / \mathrm{day})$ and long access (LgA: $6 \mathrm{~h} /$ day) in male and female rats. a Active and Inactive lever presses. b METH infusions $(0.1 \mathrm{mg} / \mathrm{kg})$ and total intake. Inset graph illustrates the difference in total METH intake between ShA and LgA rats. c Locomotor activity. d Development of binge-like METH intake in LgA trained rats (combined male and female), comparing the first and final 2 and $6 \mathrm{~h}$ sessions in 15 -minute bins. Inset graph illustrates the comparison between the first $2 \mathrm{~h}$ of the labelled sessions in LgA rats. ${ }^{\#} p<0.05$. ${ }^{*} p<0.05$ from $6 \mathrm{~h} \mathrm{D1}$. Data are presented as mean \pm SEM. $n=$ 16/sex/group.

(a)

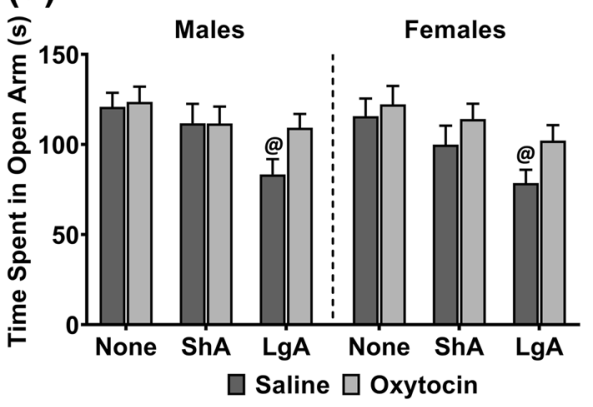

(c)

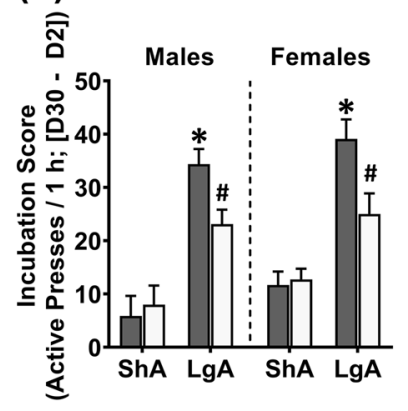

(b)

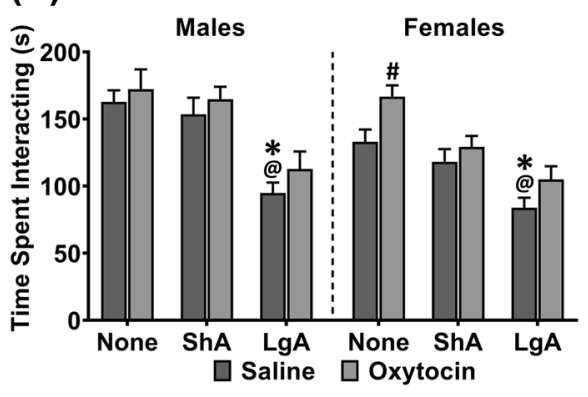

(e) (d)

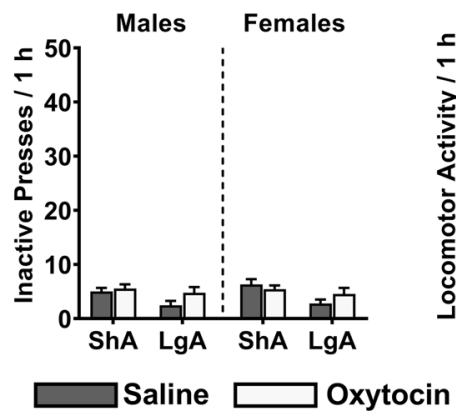

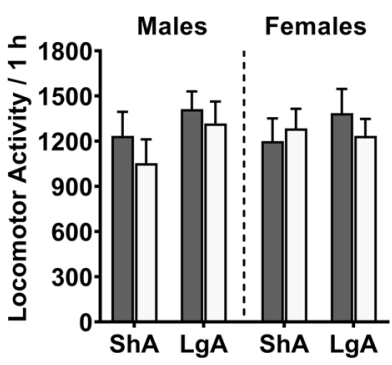

Fig. 3 The effect of chronic oxytocin treatment during abstinence on trait anxiety, social interaction, and incubation of cue-induced relapse, in male and female rats with no METH exposure ("None"), and in ShA and LgA trained rats. a Time spent in the open arms of the elevated plus maze. b Time spent interacting with a novel conspecific, c Difference in active lever presses between cue-relapse day 2 and day 30 ("Incubation Score"). d Inactive lever presses, and e locomotor activity on cue-induced relapse day $30 .{ }^{@} p<0.017$ compared with respective 'None' group. ${ }^{\#} p<0.017$ compared with respective saline group. ${ }^{*} p<0.017$ compared with respective ShA group. Data are presented as mean \pm SEM. $n=8$ per group. 
incubation scores was greater in LgA than ShA rats. There was no significant effect of Sex on incubation scores $(F(1,58)=3.836 ; p=$ $0.055)$. Comparing within Saline treated rats, LgA had significantly higher incubation scores than ShA (Males: $p<0.001$; Females: $p<$ 0.001). Comparing within LgA, oxytocin treated rats had significantly lower incubation scores than saline treated rats (Males: $p=0.012$; Females: $p=0.015$ ).

\section{Extinction}

All rats extinguished their active lever pressing within 8 extinction sessions (<15 presses; Fig. S1). There was no effect of oxytocin treatment $(F(1,58)=0.555, p=0.459)$ on the number of extinction sessions required to achieve extinction criterion. There was, however, a significant interaction of METH-Access $\times \operatorname{Sex}(\mathrm{F}(1,58)=27.198 ; p<$ $0.001)$, whereby the difference in the number of extinction sessions required to achieve extinction criterion between ShA and LgA groups was greater in males than females. LgA males required significantly more sessions to extinguish than ShA males $(p<0.001)$.

Effects of chronic oxytocin treatment on yohimbine-induced reinstatement

There was a significant effect of yohimbine dose on active lever pressing $(F(1,54)=23.158, p<0.000)$, so each dose was analysed in separate three-factor models below.

$0.625 \mathrm{mg} / \mathrm{kg}$ yohimbine. On the $0.625 \mathrm{mg} / \mathrm{kg}$ yohimbine reinstatement test, there were no significant interactions of Sex $\times$ Chronic-Treatment $\times$ METH-Access $(F(1,58)=1.478 ; \quad p=0.229)$, Sex $\times$ METH-Access $(\mathrm{F}(1,58)=2.441 ; p=0.124)$, or METH-Access $\times$ Chronic-Treatment $(F(1,58)=0.027 ; p=0.869)$ on active lever presses, while adjusting for inactive lever presses. However, there was a significant Sex $\times$ Chronic-Treatment interaction $(F(1,58)=$ 9.853; $p=0.003)$, as the difference between chronic oxytocin and saline treatment on active lever pressing was greater in females than males, regardless of METH Access. There was also a significant effect of METH-Access on active lever pressing, whereby LgA rats had higher active lever pressing than ShA rats $(\mathrm{F}(1,58)=47.859 ; p<0.001$; Fig. $4 \mathrm{a})$. There was no significant main effect of Sex $(F(1,58)=0.056 ; p=0.813)$ or Chronic-Treatment $(F(1,58)=0.995 ; p=0.323)$ on active lever pressing. Simple contrasts $(a<0.017)$ revealed that within Saline treated rats, LgA resulted in significantly higher active lever pressing than ShA (Males: $p=0.003$, Females: $p=0.004$ ). Within LgA rats, oxytocin did not significantly reduce active lever pressing compared to chronic saline treatment (Males: $p=0.226$, Females: $p=0.062$ ), whereas within ShA rats, oxytocin treatment significantly reduced active lever pressing in females $(p<0.001)$ but not males $(p=$ 0.990). Comparisons with baseline extinction data are included in the figure legends, and in Table S1.

$1.25 \mathrm{mg} / \mathrm{kg}$ yohimbine. On the $1.25 \mathrm{mg} / \mathrm{kg}$ yohimbine-induced reinstatement test, there were no significant interactions of Sex $x$ METH-Access $\times$ Chronic Treatment $(\mathrm{F}(1,58)=0.112 ; p=0.739)$ or METH-Access $\times$ Chronic Treatment $(\mathrm{F}(1,58)=0.370 ; p=0.545)$ on active lever pressing, when adjusting for inactive lever pressing. Importantly, there were significant interactions of Sex $\times \mathrm{METH}$ Access $(F(1,58)=12.343 ; p=0.001)$, as LgA induced higher active lever pressing compared to ShA more so in females than males; and Sex $\times$ Chronic Treatment $(F(1,58)=8.782 ; p=0.004)$, whereby oxytocin reduced active lever pressing compared to saline treatment to a greater extent in females than males. Simple comparisons reveal that within Saline treated rats, LgA significantly increased active lever pressing (Males: $p=0.004$, Females: $p<0.001)$. Within LgA rats, oxytocin treatment significantly reduced active lever pressing compared with saline treatment in females $(p=0.007)$ but not males $(p=0.159)$. Within ShA rats, oxytocin similarly reduced active lever pressing in females $(p=$ $0.002)$ but not males $(p=0.489)$.
Effects of chronic oxytocin treatment on methamphetamineprimed reinstatement

There was a significant effect of METH dose on active lever pressing $(F(1,54)=71.963, p<0.000)$, so each dose was analysed in separate three-factor models.

$0.3 \mathrm{mg} / \mathrm{kg}$ methamphetamine. On the $0.3 \mathrm{mg} / \mathrm{kg} \mathrm{METH}-\mathrm{primed}$ reinstatement test, there were no significant interactions of Sex $x$ Chronic-Treatment $\times$ METH-Access $(F(1,58)=0.084 ; p=0.772)$, Sex $\times$ METH-Access $(F(1,58)=0.341 ; p=0.562)$, Sex $\times$ Chronic-Treatment $(\mathrm{F}(1,58)=0.797 ; \quad p=0.376)$, or METH-Access $\times$ Chronic-Treatment $(\mathrm{F}(1,58)=1.029 ; p=0.315)$, on active lever pressing while adjusting for inactive lever pressing. There was, however, a significant effect of METH-Access on active lever pressing $(F(1,58)=13.296, p=0.001$; Fig. 5a), whereby LgA rats demonstrated significantly higher active lever pressing than ShA rats. There was no effect of Sex $(F(1,58)=$ $0.173 ; p=0.680)$ or Chronic-Treatment $(\mathrm{F}(1,58)=1.479 ; p=0.229)$. Simple contrasts within Saline treated rats found that $\mathrm{LgA}$ resulted in significantly higher active lever pressing for females $(p=0.050)$ but not males $(p=0.077)$. Within LgA rats, oxytocin compared with saline treatment did not significantly affect active lever pressing (Males: $p=0.398$, Females: $p=0.255$ ).

$1 \mathrm{mg} / \mathrm{kg}$ methamphetamine. On the $1 \mathrm{mg} / \mathrm{kg}$ METH-primed reinstatement test, there were no significant interactions of Sex $x$ METH-Access $\times$ Chronic-Treatment $\quad(F(1,58)=0.482 ; \quad p=0.490)$, Sex $\times$ METH-Access $(F(1,58)=0.227 ; p=0.636)$, Sex $\times$ Chronic-Treatment $(F(1,58)=0.449 ; p=0.506)$ on active lever pressing when adjusting for inactive lever pressing. There was a significant METHAccess $\times$ Chronic-Treatment interaction $(F(1,58)=9.011 ; p=0.004)$, whereby the difference between oxytocin and saline groups on active lever pressing was significantly greater for $\mathrm{LgA}$ than ShA rats. There was no effect of $\operatorname{Sex}(F(1,58)=0.108 ; p=0.744)$. Simple comparisons within Saline treated rats found that $\mathrm{LgA}$ significantly increased active lever pressing compared to ShA (Males: $p<0.001$, Females: $p=0.005$ ). Within LgA rats, oxytocin significantly reduced active lever pressing compared to saline (Males: $p=0.008$, Females: $p=0.010$ ). Within ShA rats, oxytocin did not significantly affect active lever pressing (Males: $p=0.075$, Females: $p=0.075$ ).

\section{DISCUSSION}

Our study investigated the effects of chronic oxytocin treatment during abstinence from METH self-administration. This data indicate that 15 days of oxytocin treatment during a 30-day abstinence period is sufficient to suppress METH relapse, and to subtly improve anxiety-like behaviours. Importantly, these findings were largely consistent between sexes, and primarily evident in rats which had extended access to METH self-administration.

Extended access to methamphetamine induced a high-addiction and high-anxiety phenotype in both sexes

The extended access procedure resulted in binge-like patterns of METH intake in LgA but not ShA rats. Bingeing is common in early stages of addiction in humans [41] and has been shown to predict several addiction-like behaviours in rodents [42]. Female LgA rats also exhibited substantially higher locomotor hyperactivity during self-administration than LgA males despite similar METH intake, which may parallel the higher rates of psychosis in METHdependent females than males [43]. Importantly, all LgA groups exhibited incubation of cue-induced relapse, and reinstated to METH more than ShA rats, while yohimbine-induced reinstatement was only elevated by $\operatorname{LgA}$ in females. This is consistent with previous demonstrations of enhanced METH-primed reinstatement in LgA compared to ShA rats [44, 45], and clinical observations of higher stress-related psychopathology in female psychostimulant users compared with males $[46,47]$. Interestingly, ShA produced a minor but significant incubation effect in females 
(a)

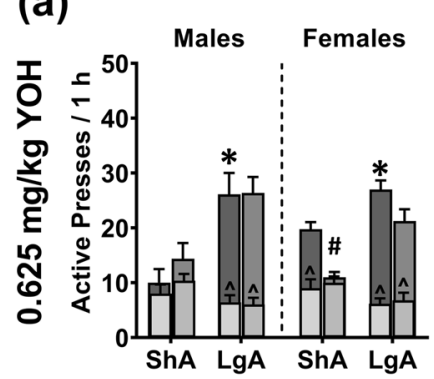

(d)

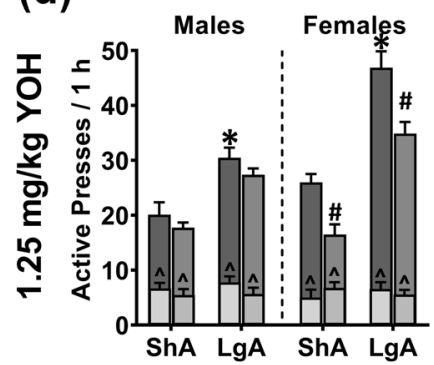

(b)

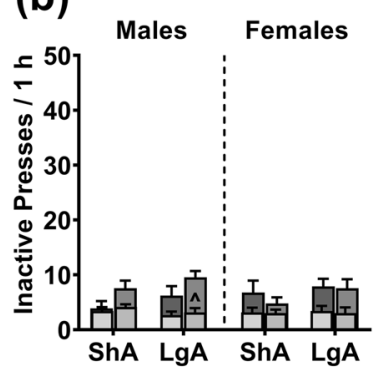

(e)

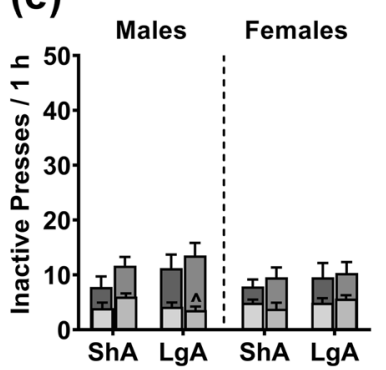

(c)

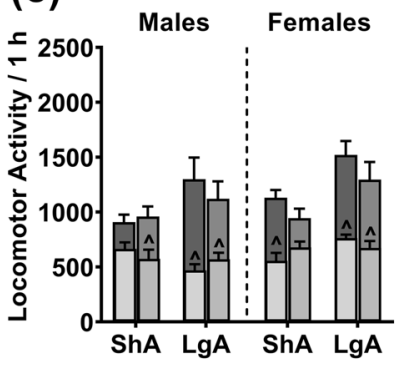

(f)

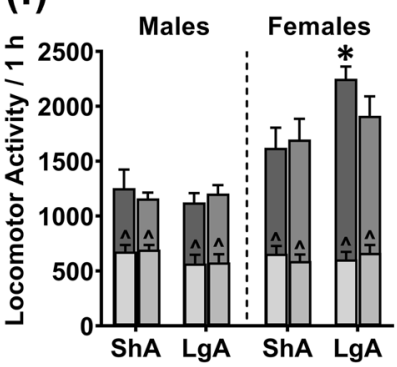

Saline $\square$ Extinction day prior

Oxytocin

\section{Extinction day prior Reinstatement test}

Fig. 4 The effect of chronic oxytocin treatment during abstinence on $0.625 \mathrm{mg} / \mathrm{kg}$ (a-c) and $1.25 \mathrm{mg} / \mathrm{kg}$ (d-f) yohimbine-primed reinstatement to METH-seeking behaviour in ShA and LgA trained male and female rats. $\mathbf{a}$, $\mathbf{d}$ Active lever presses. $\mathbf{b}$, e Inactive lever presses. c, $\mathbf{f}$ Locomotor activity. Overlaid graphs depict data from the respective extinction day prior to each test. ${ }^{*} p<0.017$ compared with respective ShA group. ${ }^{\#} p<0.017$ compared with respective saline group. ${ }^{p} p<0.017$ compared with extinction day prior to test. Data are presented as mean \pm SEM. $n=8$ /group.

(a)

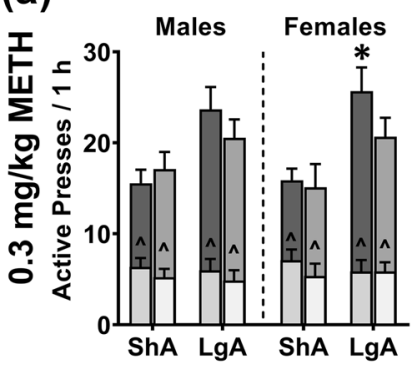

(d)

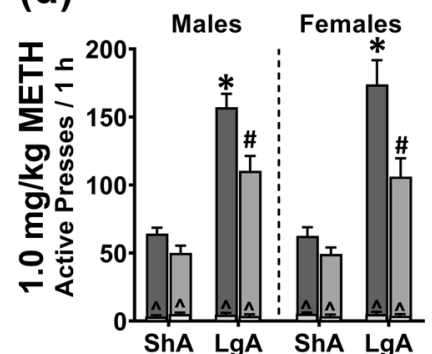

(b)

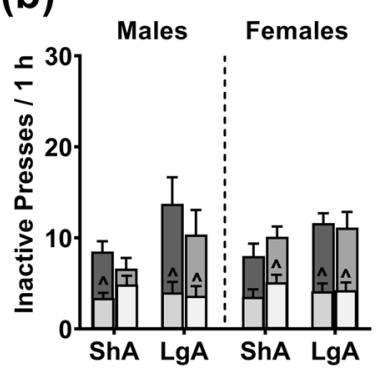

(e)

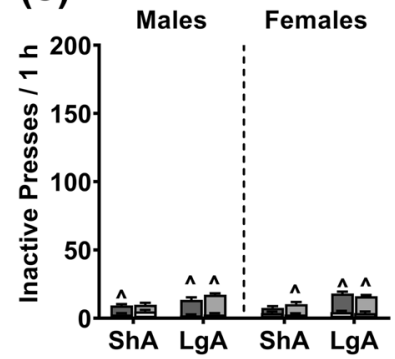

(c)

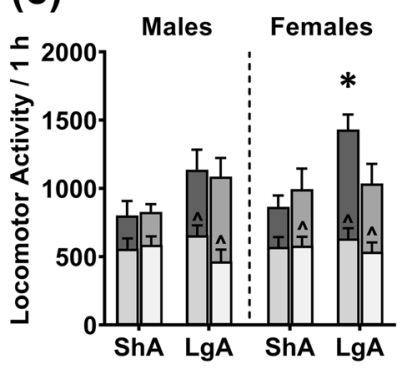

(f)

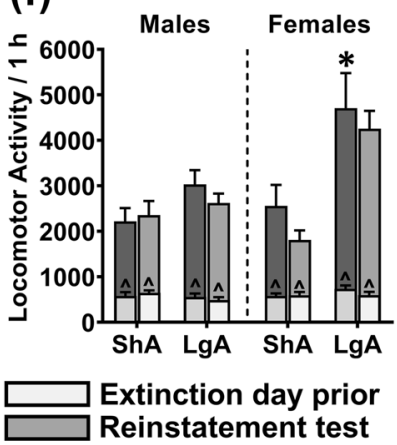

Fig. 5 The effect of chronic oxytocin treatment during abstinence on $0.3 \mathrm{mg} / \mathrm{kg}(\mathbf{a}-\mathbf{c})$ and $1.0 \mathrm{mg} / \mathrm{kg}$ (d-f) METH-primed reinstatement to METH-seeking behaviour in ShA and LgA trained male and female rats. a, d Active lever presses. b, e Inactive lever presses. c, $\mathbf{f}$ Locomotor activity. METH was administered IP immediately prior to initiation of the reinstatement session. Overlaid graphs depict data from the respective extinction day prior to each test. ${ }^{*} p<0.05$ compared with respective ShA group. ${ }^{\#} p<0.05$ compared with respective saline group. ${ }^{\wedge} p<0.05$ compared with extinction day prior to test. Data are presented as mean \pm SEM. $n=8 /$ group. 
but not males, which has not previously been shown. Otherwise, the effects of LgA on escalated METH self-administration, incubation, and METH-primed reinstatement were similar between sexes, which largely mirrors previous findings [48].

Additionally, LgA increased trait anxiety and reduced social interaction time, while ShA rats did not differ from METH-naïve controls. This is in keeping with previous reports of elevated anxiety-like behaviour on the marble burying test in LgA compared to ShA METH rats [27] and reduced social interaction time following non-contingent METH exposure [49]. Given that LgA rats do not exhibit deficits in social motivation [50], this effect of $\mathrm{LgA}$ on social interaction may reflect a heightened anxiety state, like what was measured with the EPM. Overall, this pattern of data parallels the human literature indicating high levels of anxiety in METH-dependent individuals [2,3] and supports the use of extended access procedures in both sexes to induce addiction-like phenotypes with translational validity.

Chronic oxytocin treatment during abstinence reduced relapse, reinstatement, and anxiety in extended access rats

Chronic treatment with oxytocin during abstinence from METH remediated multiple addiction-like behaviours. Firstly, oxytocin attenuated the incubation of cue-induced relapse. Incubation of METH craving after protracted abstinence critically involves activity in the central amygdala (CeA; [51]), a major recipient of hypothalamic oxytocin which inhibits CeA output [52]. As administered oxytocin stimulates endogenous oxytocin activity [53], then chronic activation of these amygdala projecting axons by IP oxytocin may have prevented the heightened CeA activity hypothesised to underpin incubation of METH craving. Interactions of oxytocin and LgA at the CeA may therefore be a fruitful pursuit.

Secondly, chronic oxytocin treatment also reduced METHprimed reinstatement in LgA but not ShA rats. Psychostimulantprimed reinstatement is driven by impaired glutamate homeostasis in fronto-striatal circuits, which is augmented by LgA [54]. Interestingly, oxytocin treatment increases expression of glutamate transporter 1 [33], which is responsible for the majority of glutamate reuptake in addiction circuits [55], and restoration of which inhibits psychostimulant-seeking more in LgA than ShA rats [56]. As such, it may be possible that oxytocin treatment worked to restore LgA-disrupted glutamate homeostasis.

Lastly, yohimbine-primed reinstatement was also attenuated by chronic oxytocin treatment, but only in females. This effect was present in both ShA and LgA females, but only LgA males, although this may be due to a floor effect on ShA males responding to yohimbine, especially at the lower dose. This is the most overt sex difference discovered in the present study and may be related to interactions between oxytocin, sex hormones, and stress. For example, yohimbine-primed reinstatement can be reduced in female but not male rats by pretreatment with allopregnanolone [57], which modulates endogenous oxytocin release [58]. Oxytocin also interacts with stress differently between sexes. For example, social defeat stress increases markers of endogenous oxytocin function in female but not male mice, while oxytocin treatment reversed behavioural responses to stress in males but not females [59]. These data suggest links between stress, oxytocin, and relapse; however, recent findings demonstrate that food-seeking induced by yohimbine requires the presence of contingent cues [60]. This indicates that yohimbine may induce reinstatement through mechanisms distinct from stress, or that stress reinstates drugseeking through augmenting the valence of cues. Given that yohimbine and foot-shock induce nicotine reinstatement through convergent neural mechanisms [61], the latter explanation may be likely. Considering this, our data may suggest that females are more susceptible to this effect on cues (e.g. ref. [38]), and that oxytocin treatment may have modulated this elevated cue-salience/valence. Such an effect of oxytocin has been hypothesised by others [62], whereby oxytocin is thought to enhance the salience of social stimuli, while dampening the salience of drug-related stimuli.

Implications for using oxytocin as an addiction therapy Across all relapse measures, chronic oxytocin treatment had a greater effect in LgA than ShA rats. This was somewhat the case for anxiety and social behaviour as well, where chronic oxytocin decreased anxiety in LgA males and females, but not in ShA or controls. These findings align with multiple studies demonstrating efficacy of oxytocin only in rodents, which exhibit dysregulated behaviour. For example, rats which have undergone a model of post-traumatic stress disorder exhibit augmented contextual fear, which is ameliorated by chronic oxytocin treatment [63]. However, non-stressed rats received minimal benefit from oxytocin, suggesting specific interference with the modelled stress pathology. Furthermore, although chronic oxytocin treatment (15 days, icv) prevented the emergence of a high-anxiety phenotype induced by chronic psychosocial stress in mice, this same chronic treatment produced a high-anxiety phenotype in non-stressed mice [64]. Clearly, the effects of oxytocin treatment on socioemotional behaviours depend upon the animal's prior history.

We hypothesise that this extends to addiction-like behaviours, as demonstrated by the differential effects of oxytocin in LgA and ShA rats shown here. One explanation for this is that METH dysregulates the endogenous oxytocin system $[65,66]$. As it has been proposed that the status of the oxytocin system may confer addiction vulnerability or susceptibility and may impact the efficacy of exogenous oxytocin $[22,67,68]$, then the efficacy of oxytocin in LgA but not ShA rats may be due to a greater LgAinduced disruption to the endogenous oxytocin system. Another possibility is that oxytocin may normalise systems disrupted by the addiction process, which are common across all drugs of abuse, rather than specific to METH. For example, acute oxytocin treatment suppresses escalated alcohol consumption and motivation in alcohol-dependent but not non-dependent rats, likely through modulating the $\mathrm{CeA}$ [69], which is also implicated in incubated METH-craving [51]. Overall, the superior therapeutic effects of oxytocin in LgA rats is particularly encouraging from a translational perspective, as LgA may better model human drug addiction (although see ref. [70])

It is largely unknown to what degree the effects of IP oxytocin administration on addiction-like behaviours are mediated by action in the periphery, which is rich in oxytocin receptors [71], or in the brain, which it is thought to poorly penetrate. Recent findings from studies using oxytocin-null mice indicate that IP oxytocin reaches the amygdala and hippocampus within $30 \mathrm{~min}$ post administration [72], and is transported into the brain by receptors for advanced glycation end-products on the blood-brain barrier [73]. Additionally, there is compelling evidence that IP oxytocin modulates feeding behaviour and oxytocin neuron activity through an ascending vagal-dependent pathway [74], and we have unpublished data indicating that oxytocin inhibits METH self-administration via the vagus nerve. With this progress in understanding the route by which IP oxytocin modulates the brain, the present findings may be interpreted with greater confidence in their potential for clinical translation.

\section{LIMITATIONS AND CONCLUSIONS}

Several limitations of this study should be considered. Firstly, oxytocin or saline was administered to pairs of rats in their home cages. As acute treatment with oxytocin induces profound social effects [75], and social bonds protect against METH selfadministration [76], it is possible that the relapse-suppressant effects of oxytocin discovered here were due to resulting differences in social bonds between the oxytocin and saline treated rats. However, as oxytocin is often used as an adjunct to 
psychosocial therapies (e.g. ref. [77]), such an effect may be clinically beneficial. Conversely, chronic oxytocin treatment may adversely alter the endogenous oxytocin system [78] and may therefore impair behaviour or cognition. However, 21 days of oxytocin (1 mg/kg IP) has shown improvement in probabilistic reversal learning without impacting effortful motivation in male rats [79], suggesting that our data are not due to impaired cognition or motivation. Lastly, estrus cycle is a potent mediator of cocaine-cue learning [38], and incubation of cocaine craving [80], indicating the importance of including estrus cycle as a variable, which we did not do. Although estrus cycle does not moderate the inhibitory effects of oxytocin on METH-primed reinstatement [7], cycle may interact with oxytocin during cue- or yohimbine-induced relapse. Understanding the sex dependent effects of oxytocin is vital for clinical translation and requires further investigation.

Overall, our findings support a relapse-prevention strategy of administering oxytocin during abstinence from METH, for both males and females. Oxytocin may also be a promising treatment for the psychosocial symptoms often comorbid with METH-use disorders. While the mechanisms by which peripherally administered oxytocin modulates addiction processes in the brain require further elucidation, this study convincingly demonstrates through the use of a translatable animal model, the clinical utility of oxytocin for treating METH-use disorders.

\section{FUNDING AND DISCLOSURE}

The project was funded by the Macquarie University Department of Psychology. NE was funded by a Research Training Program scholarship from the Australian Government. The authors have no financial interests relating to the work described and declare no conflict of interest.

\section{ACKNOWLEDGEMENTS}

The authors thank Wayne McTegg and Christine Sutter for their support in animal care throughout this long project. We also give special thanks to Dr Jean Lud Cadet for early advice on experimental design.

\section{ADDITIONAL INFORMATION}

Supplementary Information accompanies this paper at (https://doi.org/10.1038/ s41386-019-0566-6)

Publisher's note Springer Nature remains neutral with regard to jurisdictional claims in published maps and institutional affiliations.

\section{REFERENCES}

1. United Nations Office on Drugs, \& Crime. 2005 World drug report (Vol. 2). United Nations Publications; 2005.

2. Darke S, Torok M, McKetin R, Kaye S, Ross J. Patterns of psychological distress related to regular methamphetamine and opioid use. Addiction Res Theory. 2011;19:121-7.

3. Su H, Zhang J, Ren W, Xie Y, Tao J, Zhang X, et al. Anxiety level and correlates in methamphetamine-dependent patients during acute withdrawal. Medicine. 2017;96:e6434.

4. Hellem TL. A review of methamphetamine dependence and withdrawal treatment: a focus on anxiety outcomes. J Subst Abus Treat. 2016;71:16-22.

5. Morley KC, Cornish JL, Faingold A, Wood K, Haber PS. Pharmacotherapeutic agents in the treatment of methamphetamine dependence. Expert Opin Investigational Drugs. 2017;26:563-78.

6. Carson DS, Cornish JL, Guastella AJ, Hunt GE, McGregor IS. Oxytocin decreases methamphetamine self-administration, methamphetamine hyperactivity, and relapse to methamphetamine-seeking behaviour in rats. Neuropharmacology. 2010;58:38-43.

7. Cox BM, Young $A B$, See RE, Reichel CM. Sex differences in methamphetamine seeking in rats: impact of oxytocin. Psychoneuroendocrinology. 2013;38:2343-53.

8. Cox BM, Bentzley BS, Regen-Tuero H, See RE, Reichel CM, Aston-Jones G. Oxytocin acts in nucleus accumbens to attenuate methamphetamine seeking and demand. Biol Psychiatry. 2017;81:949-58.
9. Qi J, Yang JY, Wang F, Zhao YN, Song M, Wu CF. Effects of oxytocin on methamphetamine-induced conditioned place preference and the possible role of glutamatergic neurotransmission in the medial prefrontal cortex of mice in reinstatement. Neuropharmacology. 2009;56:856-65.

10. Baracz SJ, Rourke PI, Pardey MC, Hunt GE, McGregor IS, Cornish JL. Oxytocin directly administered into the nucleus accumbens core or subthalamic nucleus attenuates methamphetamine-induced conditioned place preference. Behavioural brain Res. 2012;228:185-93.

11. Everett N, Baracz S, Cornish J. Oxytocin treatment in the prelimbic cortex reduces relapse to methamphetamine-seeking and is associated with reduced activity in the rostral nucleus accumbens core. Pharmacol Biochem Behav. 2019;183:64-71.

12. Bernheim A, Leong KC, Berini $C$, Reichel CM. Antagonism of $m G l u 2 / 3$ receptors in the nucleus accumbens prevents oxytocin from reducing cued methamphetamine seeking in male and female rats. Pharmacol Biochem Behav. 2017;161:13-21.

13. Baracz SJ, Everett NA, Cornish JL. The involvement of oxytocin in the subthalamic nucleus on relapse to methamphetamine-seeking behaviour. PloS ONE 2015;10: e0136132.

14. Baracz SJ, Everett NA, McGregor IS, Cornish JL. Oxytocin in the nucleus accumbens core reduces reinstatement of methamphetamine-seeking behaviour in rats. Addiction Biol. 2016;21:316-25.

15. Everett NA, McGregor IS, Baracz SJ, Cornish JL. The role of the vasopressin V1A receptor in oxytocin modulation of methamphetamine primed reinstatement. Neuropharmacology. 2018;133:1-11.

16. Ferland $\mathrm{CL}$, Reichel $\mathrm{CM}$, McGinty JF. Effects of oxytocin on methamphetamineseeking exacerbated by predator odor pre-exposure in rats. Psychopharmacology. 2016;233:1015-24.

17. Shamay-Tsoory SG, Abu-Akel A. The social salience hypothesis of oxytocin. Biol Psychiatry. 2016;79:194-202.

18. Neumann ID, Slattery DA. Oxytocin in general anxiety and social fear: a translational approach. Biol Psychiatry. 2016;79:213-21.

19. Stauffer CS, Moschetto JM, McKernan SM, Hsiang E, Borsari B, Woolley JD. Oxytocin-enhanced motivational interviewing group therapy for methamphetamine use disorder in men who have sex with men: study protocol for a randomized controlled trial. Trials. 2019;20:145.

20. Bowen MT, Carson DS, Spiro A, Arnold JC, McGregor IS. Adolescent oxytocin exposure causes persistent reductions in anxiety and alcohol consumption and enhances sociability in rats. PloS One. 2011;6:e27237.

21. Hicks C, Cornish JL, Baracz SJ, Suraev A, McGregor IS. Adolescent pre-treatment with oxytocin protects against adult methamphetamine-seeking behavior in female rats. Addiction Biol 2016;21:304-15.

22. Baracz SJ, Everett NA, Cornish JL. The impact of early life stress on the central oxytocin system and susceptibility for drug addiction: applicability of oxytocin as a pharmacotherapy. Neurosci Biobehav Rev. 2018;pii: S0149-7634:30276-8.

23. Krasnova IN, Marchant NJ, Ladenheim B, McCoy MT, Panlilio LV, Bossert JM, et al. Incubation of methamphetamine and palatable food craving after punishmentinduced abstinence. Neuropsychopharmacology. 2014;39:2008.

24. Wang G, Shi J, Chen N, Xu L, Li J, Li P, et al. Effects of length of abstinence on decision-making and craving in methamphetamine abusers. PloS ONE. 2013;8: e68791.

25. Reichel CM, Schwendt M, McGinty JF, Olive MF, See RE. Loss of object recognition memory produced by extended access to methamphetamine self-administration is reversed by positive allosteric modulation of metabotropic glutamate receptor 5. Neuropsychopharmacology. 2011;36:782

26. Hadamitzky M, Markou A, Kuczenski R. Extended access to methamphetamine self-administration affects sensorimotor gating in rats. Behavioural Brain Res. 2011:217:386-90.

27. Jang CG, Whitfield T, Schulteis G, Koob GF, Wee S. A dysphoric-like state during early withdrawal from extended access to methamphetamine self-administration in rats. Psychopharmacology. 2013;225:753-63.

28. Schwendt M, Reichel CM, See RE. Extinction-dependent alterations in corticostriatal mGluR2/3 and mGluR7 receptors following chronic methamphetamine self-administration in rats. PLoS One. 2012;7:e34299.

29. Krasnova IN, Justinova Z, Ladenheim B, Jayanthi S, McCoy MT, Barnes C, et al. Methamphetamine self-administration is associated with persistent biochemical alterations in striatal and cortical dopaminergic terminals in the rat. PloS ONE. 2010;5:e8790.

30. Havranek T, Zatkova M, Lestanova Z, Bacova Z, Mravec B, Hodosy J, et al. Intracerebroventricular oxytocin administration in rats enhances object recognition and increases expression of neurotrophins, microtubule-associated protein 2, and synapsin I. J Neurosci Res. 2015;93:893-901.

31. Feifel D, Reza T. Oxytocin modulates psychotomimetic-induced deficits in sensorimotor gating. Psychopharmacology. 1999;141:93-98.

32. Manbeck KE, Shelley D, Schmidt CE, Harris AC. Effects of oxytocin on nicotine withdrawal in rats. Pharmacol Biochem Behav. 2014;116:84-89. 
33. Qi J, Han WY, Yang JY, Wang LH, Dong YX, Wang F, et al. Oxytocin regulates changes of extracellular glutamate and GABA levels induced by methamphetamine in the mouse brain. Addiction Biol. 2012;17:758-69.

34. Peters ST, Bowen MT, Bohrer K, McGregor IS, Neumann ID. Oxytocin inhibits ethanol consumption and ethanol-induced dopamine release in the nucleus accumbens. Addiction Biol. 2017;22:702-11.

35. Dumais KM, Kulkarni PP, Ferris CF, Veenema AH. Sex differences in neural activation following different routes of oxytocin administration in awake adult rats. Psychoneuroendocrinology. 2017;81:52-62.

36. Kogachi S, Chang L, Alicata D, Cunningham E, Ernst T. Sex differences in impulsivity and brain morphometry in methamphetamine users. Brain Struct Funct. 2017;222:215-27.

37. Venniro M, Caprioli D, Shaham Y. Animal models of drug relapse and craving: from drug priming-induced reinstatement to incubation of craving after voluntary abstinence. Prog brain Res. 2016;224:25-52.

38. Johnson AR, Thibeault KC, Lopez AJ, Peck EG, Sands LP, Sanders CM, et al. Cues play a critical role in estrous cycle-dependent enhancement of cocaine reinforcement. Neuropsychopharmacology. 2019;44:1189.

39. Moran-Santa Maria MM, McRae-Clark A, Baker NL, Ramakrishnan V, Brady KT Yohimbine administration and cue-reactivity in cocaine-dependent individuals. Psychopharmacology 2014;231:4157-65.

40. Shepard JD, Bossert JM, Liu SY, Shaham Y. The anxiogenic drug yohimbine reinstates methamphetamine seeking in a rat model of drug relapse. Biol Psychiatry. 2004;55:1082-9.

41. Koob GF, Volkow ND. Neurocircuitry of addiction. Neuropsychopharmacology. 2010;35:217.

42. Belin D, Balado E, Piazza PV, Deroche-Gamonet V. Pattern of intake and drug craving predict the development of cocaine addiction-like behavior in rats. Biol psychiatry. 2009;65:863-8.

43. Mahoney JJ III, Hawkins RY, De La Garza R II, Kalechstein AD, Newton TF. Relationship between gender and psychotic symptoms in cocaine-dependent and methamphetamine-dependent participants. Gend Med. 2010;7:414-21.

44. Rogers JL, De Santis S, See RE. Extended methamphetamine self-administration enhances reinstatement of drug seeking and impairs novel object recognition in rats. Psychopharmacology. 2008;199:615.

45. Schwendt M, Rocha A, See RE, Pacchioni AM, McGinty JF, Kalivas PW. Extended methamphetamine self-administration in rats results in a selective reduction of dopamine transporter levels in the prefrontal cortex and dorsal striatum not accompanied by marked monoaminergic depletion. J Pharmacol Exp Therapeutics. 2009;331:555-62.

46. Fox HC, Sinha R. Sex differences in drug-related stress-system changes: implications for treatment in substance-abusing women. Harv Rev psychiatry. 2009;17:103-19.

47. Salo R, Flower K, Kielstein A, Leamon MH, Nordahl TE, Galloway GP. Psychiatric comorbidity in methamphetamine dependence. Psychiatry Res. 2011;186:356-61.

48. Venniro M, Zhang M, Shaham Y, Caprioli D. Incubation of methamphetamine but not heroin craving after voluntary abstinence in male and female rats. Neuropsychopharmacology. 2017;42:1126.

49. Clemens KJ, Van Nieuwenhuyzen PS, Li KM, Cornish JL, Hunt GE, McGregor IS. MDMA ("ecstasy"), methamphetamine and their combination: long-term changes in social interaction and neurochemistry in the rat. Psychopharmacology. 2004;173:318-25.

50. Venniro M, Zhang M, Caprioli D, Hoots JK, Golden SA, Heins C, et al. Volitiona social interaction prevents drug addiction in rat models. Nat Neurosci. 2018;21:1520.

51. Li X, Zeric T, Kambhampati S, Bossert JM, Shaham Y. The central amygdala nucleus is critical for incubation of methamphetamine craving. Neuropsychopharmacology. 2015;40:1297.

52. Knobloch HS, Charlet A, Hoffmann LC, Eliava M, Khrulev S, Cetin AH, et al. Evoked axonal oxytocin release in the central amygdala attenuates fear response. Neuron. 2012;73:553-66.

53. Carson DS, Hunt GE, Guastella AJ, Barber L, Cornish JL, Arnold JC, et al. Systemically administered oxytocin decreases methamphetamine activation of the subthalamic nucleus and accumbens core and stimulates oxytocinergic neurons in the hypothalamus. Addiction Biol. 2010;15:448-63.

54. J Roberts-Wolfe D, W Kalivas. Glutamate transporter GLT-1 as a therapeutic target for substance use disorders. CNS Neurological Disord-Drug Targets. 2015;14:745-56.

55. Danbolt NC. Glutamate uptake. Prog Neurobiol. 2001;65:1-105.

56. Fischer KD, Houston AC, Rebec GV. Role of the major glutamate transporter GLT1 in nucleus accumbens core versus shell in cue-induced cocaine-seeking behavior. J Neurosci. 2013;33:9319-27.

57. Anker JJ, Carroll ME. Sex differences in the effects of allopregnanolone on yohimbine-induced reinstatement of cocaine seeking in rats. Drug Alcohol Depend. 2010;107:264-7.
58. Blyth BJ, Hauger RL, Purdy RH, Amico JA. The neurosteroid allopregnanolone modulates oxytocin expression in the hypothalamic paraventricular nucleus. Am J Physiol-Regulatory, Integr Comp Physiol. 2000;278:R684-91.

59. Steinman MQ, Duque-Wilckens N, Greenberg GD, Hao R, Campi KL, Laredo SA, et al. Sex-specific effects of stress on oxytocin neurons correspond with responses to intranasal oxytocin. Biol Psychiatry. 2016;80:406-14.

60. Chen YW, Fiscella KA, Bacharach SZ, Tanda G, Shaham Y, Calu DJ. Effect of yohimbine on reinstatement of operant responding in rats is dependent on cue contingency but not food reward history. Addiction Biol. 2015;20:690-700.

61. Nygard SK, Hourguettes NJ, Sobczak GG, Carlezon WA, Bruchas MR. Stressinduced reinstatement of nicotine preference requires dynorphin/kappa opioid activity in the basolateral amygdala. J Neurosci. 2016;36:9937-48.

62. Bowen MT, Neumann ID. Rebalancing the addicted brain: oxytocin interference with the neural substrates of addiction. Trends Neurosci. 2017;40:691-708.

63. Janezic EM, Uppalapati S, Nagl S, Contreras M, French ED, Fellous JM. Beneficial effects of chronic oxytocin administration and social co-housing in a rodent model of post-traumatic stress disorder. Behavioural Pharmacol. 2016;27:704-17.

64. Peters S, Slattery DA, Uschold-Schmidt N, Reber SO, Neumann ID. Dosedependent effects of chronic central infusion of oxytocin on anxiety, oxytocin receptor binding and stress-related parameters in mice. Psychoneuroendocrinology. 2014;42:225-36.

65. Baracz SJ, Parker LM, Suraev AS, Everett NA, Goodchild AK, McGregor IS, et al. Chronic methamphetamine self-administration dysregulates oxytocin plasma levels and oxytocin receptor fibre density in the nucleus accumbens core and subthalamic nucleus of the rat. J Neuroendocrinol. 2016;28. https://onlinelibrary. wiley.com/doi/full/10.1111/jne.12337.

66. Georgiou P, Zanos P, Garcia-Carmona JA, Hourani S, Kitchen I, Laorden ML, et al. Methamphetamine abstinence induces changes in $\mu$-opioid receptor, oxytocin and CRF systems: Association with an anxiogenic phenotype. Neuropharmacology. 2016;105:520-32

67. Buisman-Pijlman FT, Sumracki NM, Gordon JJ, Hull PR, Carter CS, Tops M. Individual differences underlying susceptibility to addiction: role for the endogenous oxytocin system. Pharmacol Biochem Behav. 2014;119:22-38.

68. Bowen MT, Liu J, Buisman-Pijlman FT. Oxytocin: providing new avenues for treating and understanding problematic drug use. Neuropathol Drug Addict Subst Misuse. 2018;3:82-92.

69. Tunstall BJ, Kirson D, Zallar LJ, McConnell SA, Vendruscolo JC, Ho CP, et al Oxytocin blocks enhanced motivation for alcohol in alcohol dependence and blocks alcohol effects on GABAergic transmission in the central amygdala. PLoS Biol. 2019;17:e2006421.

70. Cadet JL. Animal models of addiction: compulsive drug taking and cognition Neurosci Bio-behav Rev. 2019;106:5-6. https://www.ncbi.nlm.nih.gov/pubmed/ 31176644.

71. Jard S, Barberis C, Audigier S, Tribollet E. Neurohypophyseal hormone receptor systems in brain and periphery. Prog Brain Res. 1987;72:173-87.

72. Smith AS, Korgan AC, Young WS. Oxytocin delivered nasally or intraperitoneally reaches the brain and plasma of normal and oxytocin knockout mice. Pharmacol Res. 2019;146:104324.

73. Yamamoto $Y$, Liang M, Munesue S, Deguchi K, Harashima A, Furuhara K, et al. Vascular RAGE transports oxytocin into the brain to elicit its maternal bonding behaviour in mice. Communications. Biology. 2019;2:76.

74. Iwasaki Y, Kumari P, Wang L, Hidema S, Nishimori K, Yada T. Relay of peripheral oxytocin to central oxytocin neurons via vagal afferents for regulating feeding. Biochemical Biophysical Res Commun. 2019;519:553-8.

75. Ramos L, Hicks C, Kevin R, Caminer A, Narlawar R, Kassiou M, et al. Acute prosocial effects of oxytocin and vasopressin when given alone or in combination with 3 , 4-methylenedioxymethamphetamine in rats: involvement of the $\mathrm{V} 1 \mathrm{~A}$ receptor. Neuropsychopharmacology. 2013;38:2249.

76. Westenbroek C, Perry AN, Jagannathan L, Becker JB. Effect of social housing and oxytocin on the motivation to self-administer methamphetamine in female rats. Physiol Behav. 2017;203:10-17.

77. Guastella AJ, Howard AL, Dadds MR, Mitchell P, Carson DS. A randomized controlled trial of intranasal oxytocin as an adjunct to exposure therapy for social anxiety disorder. Psychoneuroendocrinology. 2009;34:917-23.

78. Jurek B, Neumann ID. The oxytocin receptor: from intracellular signaling to behavior. Physiological Rev. 2018;98:1805-908.

79. Roberts BZ, Young JW, He YV, Cope ZA, Shilling PD, Feifel D. Oxytocin improves probabilistic reversal learning but not effortful motivation in Brown Norway rats. Neuropharmacology. 2019;150:15-26.

80. Nicolas C, Russell TI, Pierce AF, Maldera S, Holley A, You ZB, et al. Incubation of cocaine craving after intermittent-access self-administration: Sex differences and estrous cycle. Biol Psychiatry. 2019;85:915-24. 